\title{
Hepatic Rupture Complicating HELLP Syndrome and Pre-existent Antiphospholipid Syndrome with Cerebral Venous Sinus Thrombosis
}

\section{Chinmayi Patkar-Kattimani ${ }^{1 *}$, Riddhi Rathod ${ }^{1}$, Dinesh Sagtani ${ }^{2}$ and Prasad Kattimani ${ }^{1}$}

${ }^{1}$ Specialty Doctor, Department of Anaesthesia and Intensive Care, Queen's Hospital, Barking, Havering and Redbridge University Hospitals NHS Trust, Romford, Essex, United Kingdom

${ }^{2}$ Consultant, Department of Anaesthesia and Intensive Care, Queen's Hospital, Barking, Havering and Redbridge University Hospitals NHS Trust, Romford, Essex, United Kingdom

*Corresponding Author: Chinmayi Patkar-Kattimani, Specialty Doctor, Department of Anaesthesia and Intensive Care, Queen's Hospital, Barking, Havering and Redbridge University Hospitals NHS Trust, Romford, Essex, United Kingdom.

DOI: $10.31080 /$ ASPS.2020.04.0572
Received: June 08, 2020

Published: July 28, 2020

(C) All rights are reserved by Chinmayi

Patkar-Kattimani., et al.

\section{Abstract}

Antiphospholipid syndrome is associated with HELLP (Haemolysis, Elevated Liver enzymes and Low Platelets) syndrome as a continuum of thrombotic microangiopathies. Subcapsular liver haematoma is a rare presentation of HELLP syndrome. A 38-year-old woman at 33 weeks gestation presented with HELLP syndrome complicated by acute hepatic rupture. She had background history of antiphospholipid syndrome with cerebral venous sinus thrombosis. Postoperative course was complicated by hepatic failure, sepsis and haemodynamic instability which was managed successfully. Anaesthetists need to be aware of serious complications like haemorrhage, thrombosis and multi-organ failure when presented with HELLP syndrome and antiphosholipid syndrome.

Keywords: HELLP Syndrome; Antiphospholipid Syndrome; Cerebral Venous Sinus Thrombosis; Subcapsular Liver Haematoma; Hepatic Rupture

\section{Introduction}

HELLP syndrome comprises haemolysis, elevated liver enzymes and low platelet count which complicates 0.5 to $0.9 \%$ of all pregnancies and $10-20 \%$ of cases with severe preeclampsia [1]. Antiphospholipid syndrome (APS) is an autoimmune, hypercoagulable condition caused by autoantibodies to membrane phospholipid resulting in multisystem involvement. Previous case reports have implied that HELLP syndrome is more aggressive and accompanied with serious complications when associated with APS [2]. We describe HELLP syndrome associated with antiphospholipid syndrome and cerebral venous sinus thrombosis (CVST) at 33 weeks gestation which culminated in hepatic rupture. Case was appropriately managed by a multidisciplinary team with good outcome.

\section{Case Report}

A 38-year-old woman (gravida 6 para 1 abortus 4) presented to maternity triage at 33 weeks gestation with severe midsternal chest pain for six hours. The pain was continuous, severe sharp stabbing in nature and radiating to the right shoulder. It was associated with vague epigastric and lower abdominal pain and mild nausea. The patient was restless due to severe pain but denied any headache. She was intermittently dizzy, however responded appropriately to verbal commands. 
She reported a background history of CVST suffering from recurrent severe headaches. She had been investigated for unfavorable obstetric history of recurrent miscarriages and was diagnosed with APS. Past surgical history included an emergency caesarean section for preeclampsia. Her current medication was subcutaneous enoxaparin $60 \mathrm{mg}$ BD and tablet aspirin $75 \mathrm{mg}$ OD.

Immediate medical examination and vital monitoring revealed apyrexia, heart rate 100 beats per minute, blood pressure 140/90 $\mathrm{mmHg}$ and respiratory rate 18 per minute. Her neurological examination was normal with Glasgow Coma scale 15/15 and normal reflexes. Chest was clear on auscultation and abdomen was soft without any signs of guarding, tenderness and rigidity. Foetal heart rate was normal and cardiotocograph was unremarkable.

Full blood count demonstrated haemoglobin $123 \mathrm{gm} / \mathrm{L}$, platelets $96 \times 10^{9} / \mathrm{L}$ and white cell count $121 \times 10^{9} / \mathrm{L}$. Liver function tests including coagulation were normal except for a raised alanine transaminase (ALT) of $147 \mathrm{U} / \mathrm{L}$. Arterial blood gas was unremarkable. Urine protein was raised $0.72 \mathrm{gm} / \mathrm{L}$ with a borderline proteincreatinine ratio (PCR) of 36.2. Chest X-ray showed no abnormality and electrocardiogram suggested sinus tachycardia. A presumptive diagnosis of preeclampsia with HELLP syndrome was made while maintaining a clinical suspicion of intracranial haemorrhage or pulmonary embolism. An urgent head CT-scan was performed which demonstrated no abnormality. Immediately following transfer of patient from the CT room to labour ward, there was a sudden drop in blood pressure to $84 / 44 \mathrm{mmHg}$ followed by foetal bradycardia.

She was rushed to the operation theatre for category I caesarean section. Standard monitors were applied on her. $0.3 \mathrm{M}$ sodium citrate was given orally just before induction of anaesthesia. Rapid sequence induction with cricoid pressure was performed using 400 mg thiopental and $100 \mathrm{mg}$ succinylcholine. Trachea was intubated with size $7.0 \mathrm{~mm}$ internal diameter single lumen endotracheal tube after visualising the larynx with videolaryngoscope. Anaesthesia was maintained with sevoflurane in oxygen and air. Atracurium was used to maintain muscle relaxation to facilitate surgery. Right radial artery was cannulated for invasive blood pressure monitoring after induction. A live baby was delivered (APGAR score 8, 9 at 1, 5 minute) and transferred to neonatal intensive care unit (NICU) for further observation. Oxytocin 5 Units bolus was given followed by 40 units in $500 \mathrm{~mL}$ normal saline infusion as a standard practice of unit.

The surgeons noted massive intraabdominal haemorrhage and detected a large anterior subcapsular liver haematoma (Figure 1). Injection tranexamic acid 1 gram was administered. Metaraminol infusion was started to maintain mean arterial pressure above 65 $\mathrm{mmHg}$. Intraperitoneal lavage and peri hepatic packing with roller gauze were done to achieve compression haemostasis. The estimated blood loss was $1600 \mathrm{~mL}$ and patient was transfused with two units of packed red cells and two units of fresh frozen plasma. Patient was electively ventilated and transferred to the intensive therapy unit (ITU) on propofol and remifentanil infusion. The plan was to return to operation theatre the next day for removal of abdominal pack. The next twelve hours showed rapid deterioration in liver function with ALT rising up to 3277 IU/L, lactate increased to $7 \mathrm{mmol} / \mathrm{L}$ with a fall in haemoglobin and platelets (Table 1). Patient remained haemodynamically unstable requiring noradrenaline and vasopressin infusion for cardiovascular support in the ITU.

\begin{tabular}{|l|c|c|c|c|c|c|c|}
\hline & Baseline & 4 hours & $\mathbf{1 0}$ hours & $\mathbf{1 4}$ hours & $\mathbf{1 8}$ hours & 24 hours & 36 hours \\
\hline $\mathrm{Hb}(\mathrm{gm} / \mathrm{L})$ & 123 & 104 & 106 & 86 & 81 & 72 & 85 \\
\hline $\mathrm{WBC}\left(\times 10^{9} / \mathrm{L}\right)$ & 12.1 & 17.7 & 17.6 & 17.4 & 22.3 & 18.4 & 19.0 \\
\hline Platelets $\left(\times 10^{9} / \mathrm{L}\right)$ & 96 & 71 & 114 & 102 & 107 & 110 & 89 \\
\hline $\mathrm{CRP}(\mathrm{mg} / \mathrm{L})$ & & 18 & 29 & & 48 & 97 & 115 \\
\hline PT/APTT (s) & $10.0 / 28.9$ & $10.9 / 32$ & $12 / 26.6$ & & $14.2 / 31.2$ & $14.5 / 36.3$ & $13.9 / 32.6$ \\
\hline ALT (IU/L) & 142 & 259 & 998 & 1944 & 3277 & 4199 & 4317 \\
\hline Bilirubin & 6 & 44 & 48 & 26 & 17 & & \\
\hline Creatinine & 44 & 38 & 70 & 97 & 80 & & 67 \\
\hline Lactate (mmol/L) & & & 3.8 & & 7.0 & & \\
\hline
\end{tabular}

Table 1: Laboratory investigations during hospital course. 


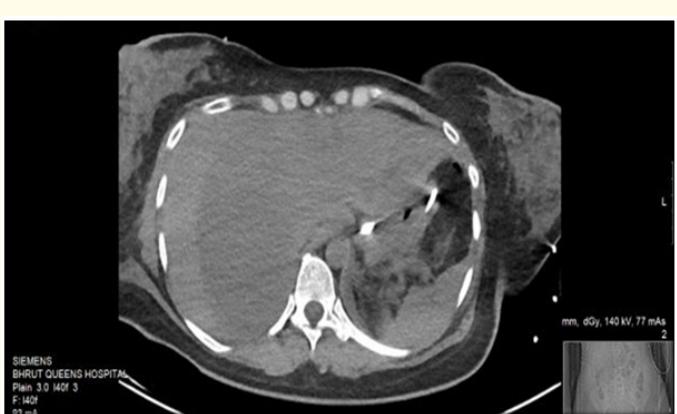

Figure 1: CT-scan abdomen: Right lobe liver subcapsular haematoma.

She returned to operation theatre for abdominal pack removal thirty-six hours after the caesarean section. On exploration, the left lobe of liver was found to be pink and healthy, but the right lobe was blue and necrotic. The liver surface was again packed, and abdomen closed. The case was discussed with hepatobiliary team at a tertiary hospital and the patient was transferred under their care on second postoperative day. At the specialist centre, patient was taken to operation theatre for a second re-look laparotomy and the abdominal pack was removed. She underwent argon LASER treatment of the hepatic capsular tear. She received continual ventilatory and haemodynamic support in ITU. She was enlisted on a standby list for hepatectomy and liver transplantation. By fourth postoperative day, patient's liver function started to improve gradually, there were no signs of clinical deterioration or bleeding noted and she did not require any further surgical intervention. The inotropic support was slowly weaned off and patient was successfully extubated on the fifth postoperative day. She was transferred back to our hospital on the seventh postoperative day.

\section{Discussion}

APS is characterized by arterial and venous thromboses, thrombocytopenia, recurrent miscarriages and neurological events. APS without any underlying medical condition is termed as primary APS whereas secondary APS is associated with an autoimmune disorder, mostly systemic lupus erythematosus [2]. Primary APS rarely presents as cerebral venous sinus thrombosis (CVST). Clinical manifestations of CVST are headaches, seizures, altered consciousness, and neurological focal signs $[3,4]$.

APS results in higher occurrence of early-onset pre-eclampsia, eclampsia and HELLP syndrome along with adverse foetal out- comes. Incidence of HELLP syndrome in APS patients is difficult to determine; however it is more severe and occurs earlier in pregnancy than in patients not affected by APS [5]. The rate of APS in patients with HELLP syndrome was $10.5 \%$ in a prospective study by Le Thi Thoung., et al. [2,5].

Catastrophic antiphospholipid antibody syndrome (CAPS) is a fatal variant of APS which poses a diagnostic and therapeutic dilemma. It has a prevalence of $1 \%$ in APS population and often leads to rapidly progressive multi-organ failure [6,7]. Severe HELLP syndrome with single organ thrombosis has been defined as 'CAPSlike' disease, as these patients warrant close monitoring for the development of CAPS and may require aggressive management similar to CAPS [7]. Women with APS and previous thrombosis with or without pregnancy morbidity are commonly treated with low dose aspirin plus therapeutic dose of heparin which was already ongoing in our patient. Additional treatments under evaluation include intravenous immunoglobulin infusions, plasma exchange and low-dose steroids [8].

The clinical spectrum of HELLP syndrome ranges from a mild and self-limiting disease to fulminant, life-threatening complications culminating in multiorgan failure [9]. Hepatic rupture is a complex critical complication of HELLP syndrome, others include disseminated intravascular coagulation (DIC), acute respiratory distress syndrome (ARDS) and renal failure.

Spontaneous rupture of subcapsular liver haematoma in pregnancy is a rare, but near-fatal incident that occurs 1 in 40,000 to 1 in 250,000 and in 1 to $<2 \%$ cases of HELLP syndrome [10]. It carries a high maternal mortality rate from $18 \%$ to $86 \%$ and perinatal mortality rate of up to $80 \%$ [11]. Hepatic rupture presents as a medical emergency and needs urgent attention to prevent any further haemodynamic deterioration. The non-specific signs and symptoms at presentation namely nausea, vomiting and right epigastric pain as observed in our case obscure an early diagnosis and rapid treatment. Diagnostic criteria for HELLP syndrome in the Tennessee classification system are LDH > $600 \mathrm{U} / \mathrm{L}, \mathrm{AST} \geq 70 \mathrm{U} / \mathrm{L}$, and platelets $<100 \times 10^{9} / \mathrm{L}$ which were fulfilled in our case [12].

Management of subcapsular liver haematoma and rupture includes interventions such as surgical exploration, perihepatic packing, haematoma evacuation, hepatic artery embolization or ligation and liver resection. Haemodynamically stable patients with HELLP associated hepatic rupture can be managed conservatively [13]. 
However, patients with complicated HELLP syndrome are best managed at a centre with expertise in liver transplantation [14].

Anaesthetic experience of HELLP syndrome with antiphospholipid syndrome and eclampsia for caesarean section at 23 weeks gestation has been described by Jo., et al. [15]. Balanced general anaesthesia with appropriate haemodynamic monitoring was fundamental in our patient management. Meticulous clinical assessments in ITU and laboratory follow-ups helped to accomplish a well-timed referral and patient transfer to the specialist hepatobiliary centre.

We suggest that the possibility of CAPS or HELLP syndrome complicating APS in pregnancy should always be borne in mind in order to prevent subsequent adverse consequences. Anaesthetic plan must be adapted suitably to help cope with the potential problems of hepatic failure such as haemodynamic instability and coagulopathy.

\section{Conclusion}

This case proved to be challenging because pre-existing APS and CVST were complicated by new-onset HELLP syndrome which eventually led to hepatic rupture. Timely diagnosis and treatment with a multidisciplinary approach was the key to successful management with favourable maternal and foetal outcomes.

\section{Conflict of Interests}

None.

\section{Acknowledgement}

The patient gave her written consent for the publication of this case report.

\section{Bibliography}

1. Haram K., et al. "The HELLP syndrome: clinical issues and management. A Review". BMC Pregnancy Childbirth 9.1 (2009): 8.

2. A Tufano., et al. "HELLP Syndrome and Its Relation with the Antiphospholipid Syndrome”. Vox Sanguinis 12.1 (2014): 114118.

3. Luo Y., et al. "Diagnosis and Treatment of Cerebral Venous Thrombosis: A Review". Frontier Aging Neuroscience 10 (2018): 2.

4. Dag ZO., et al. "HELLP Syndrome and Cerebral Venous Sinus Thrombosis Associated with Factor V Leiden Mutation during Pregnancy". Case Reports in Obstetrics and Gynecology (2014): 582890.
5. Thuong D., et al. "The HELLP Syndrome in the Antiphospholipid Syndrome: Retrospective Study of 16 Cases in 15 Women". Annals of the Rheumatic Diseases 64.2 (2005): 273-278.

6. Kazzaz NM., et al. "Treatment of catastrophic antiphospholipid syndrome". Current Opinion on Rheumatology 28.3 (2016): 218-227.

7. Aguiar CL and Erkan D. "Catastrophic antiphospholipid syndrome: how to diagnose a rare but highly fatal disease". Therapeutic Advances in Musculoskeletal Disease 5.6 (2013): 305314.

8. Radu A., et al. "Pregnancy Management in Women with Antiphospholidic Syndrome". Maedica (Buchar) 14.2 (2019): 148-160.

9. Kapan M., et al. "Subcapsular Liver Hematoma in HELLP Syndrome: Case Report”. Gastroenterology Research 3.3 (2010): 144-146.

10. Troja A., et al. "Management of Spontaneous Hepatic Rupture on Top of HELLP Syndrome: Case Report and Review of the Literature". Viszeralmedizin 31.3 (2015): 205-208.

11. Mikolasevic I., et al. "Liver Disease During Pregnancy: A Challenging Clinical Issue”. Medical Science Monitor 24 (2018): 4080-4090.

12. Haram K., et al. "The HELLP syndrome: clinical issues and management. A Review”. BMC Pregnancy Childbirth 26.9 (2009): 8.

13. Wilson SG., et al. "The management of the surgical complications of HELLP syndrome". Annals of the Royal College of Surgeons of England 96.7 (2014): 512-516.

14. Shames BD., et al. "Liver transplantation for HELLP syndrome". Liver Transplantation 11 (2005): 224-228.

15. Jo YY., et al. "Anesthetic management of HELLP syndrome complicating primary antiphospholipid syndrome -A case report". Korean Journal of Anesthesiology 62.6 (2012): 575-578.

\section{Assets from publication with us}

- Prompt Acknowledgement after receiving the article

- Thorough Double blinded peer review

- Rapid Publication

- Issue of Publication Certificate

- High visibility of your Published work

Website: www.actascientific.com/

Submit Article: www.actascientific.com/submission.php

Email us: editor@actascientific.com

Contact us: +919182824667 